\title{
Design and radiation tests on a LED based emergency evacuation directional lighting
}

\author{
Juan Casas \\ CERN-TE/CRG
}

1211 Geneva 23, Switzerland

E-mail: juan.casas-cubillos@cern.ch

\section{Nikolaos Trikoupis ${ }^{1}$}

CERN-TE/CRG

1211 Geneva 23, Switzerland

E-mail:n.trikoupis@cern.ch

\begin{abstract}
A LED (Light Emitting Diode) based directional lighting system has been designed to indicate the best evacuation direction for applications like the Large Hadron Collider (LHC) tunnel. The design includes constraints for redundancy required by safety systems and for components selection by radiation effects. Most of the literature for radiation effects on LEDs concern digital communications systems, although some recent reports do exist for visible spectrum power LEDs and the reduction in light output versus dose is coherent with the results presented in this paper. Prototype lighting units were irradiated in CERN's CHARM facility up to a Total Integrated Dose (TID) of 870 Gy and no failures were observed. This paper describes the basic design, presents field tests and the effects of radiation on the LEDs luminance.
\end{abstract}

Topical Workshop on Electronics for Particle Physics

11 - 14 September 2017

Santa Cruz, California

\section{${ }^{1}$ Speaker}




\section{Introduction}

Directional lighting that guides the personnel present in confined spaces would be a useful addition for evacuation procedures, however the authors were not able to find a manufacturer that provides such equipment qualified to operate in a radiation environment. The use of directional lighting was investigated during the tests performed in 2014 simulating an accidental liquid helium release [1] into the $27 \mathrm{~km}$ circumference LHC accelerator tunnel that lies about $100 \mathrm{~m}$ below ground level and has 9 access shafts. The LHC safety system provides, in its present status, excellent protection to the personnel present in the tunnel although a directional lightning system would be a useful addition to avoid personnel going initially towards the wrong exit shaft when an evacuation alarm is triggered.

\section{Directional Lighting Design}

The directional lighting uses red and green lights to forbid or permit circulation in a given direction inside the tunnel. The maximum separation between the lights is limited by the tunnel curvature that has a maximum line of sight of about $320 \mathrm{~m}$ although typical locations for personnel and equipment will limit it to less than $220 \mathrm{~m}$. The directional lightning shall satisfy the contradictory requirements of being visible over $350 \mathrm{~m}$ and of limiting the amount of glare while working in close proximity

\subsection{Main Components}

The light emitting elements are red and green LEDs XLamp® XP-E2 model manufactured by Cree®. The LED light is guided using a lens model FCN12790_IRIS-M manufactured by Ledil, made of PMMA (Polymethyl methacrylate) material and with a Full Width Half Maximum (FWHM) angle of $29^{\circ}$.

At the nominal LED current of $350 \mathrm{~mA}$, the red and green LEDs produce an approximate luminous flux of respectively $60 \mathrm{~lm}$ and $100 \mathrm{~lm}$. These luminous flux through the IRIS lens would be far too bright resulting in glare that exceeds the upper vision tolerance threshold (approximately $300^{\prime} 000 \mathrm{~cd} / \mathrm{m}^{2}$ ) and within distances of $5 \mathrm{~m}$ or less, may be dangerous to personnel working in close proximity to the light source [2].

To address glare, the XLamp LEDs are used with a very strong derating (current of $10 \mathrm{~mA}$ and $5 \mathrm{~mA}$ respectively for the red and green LED) and by using per color up to five Ledil IRIS lenses in parallel to increase the light emittance area to about $57 \mathrm{~cm}^{2}$. In these conditions the luminance measured at the lens surface is below $10^{\prime} 000 \mathrm{~cd} / \mathrm{m}^{2}$, this value is acceptable to personel working nearby and is within the range found for traffic red lights [3].

\subsection{Tunnel and Radiation Constraints}

The relatively low currents permit to design a robust radiation scheme for which the safety control equipment and dc power supply stays in a radiation-protected area and only passive components (resistors and diodes) are located in the hazardous area (Fig. 1).

In an application like the LHC, distances up to $2 \mathrm{~km}$ may exist between the radiation protected zone and the directional lighting. The associated cable resistance (up to $150 \mathrm{ohm}$ assuming copper cables with a cross-section of $0.5 \mathrm{~mm} 2$ ) produces a maximum voltage drop of $1.5 \mathrm{Vdc}$ which, from practical considerations, is very low compared to the $48 \mathrm{Vdc}$ supply installed. 

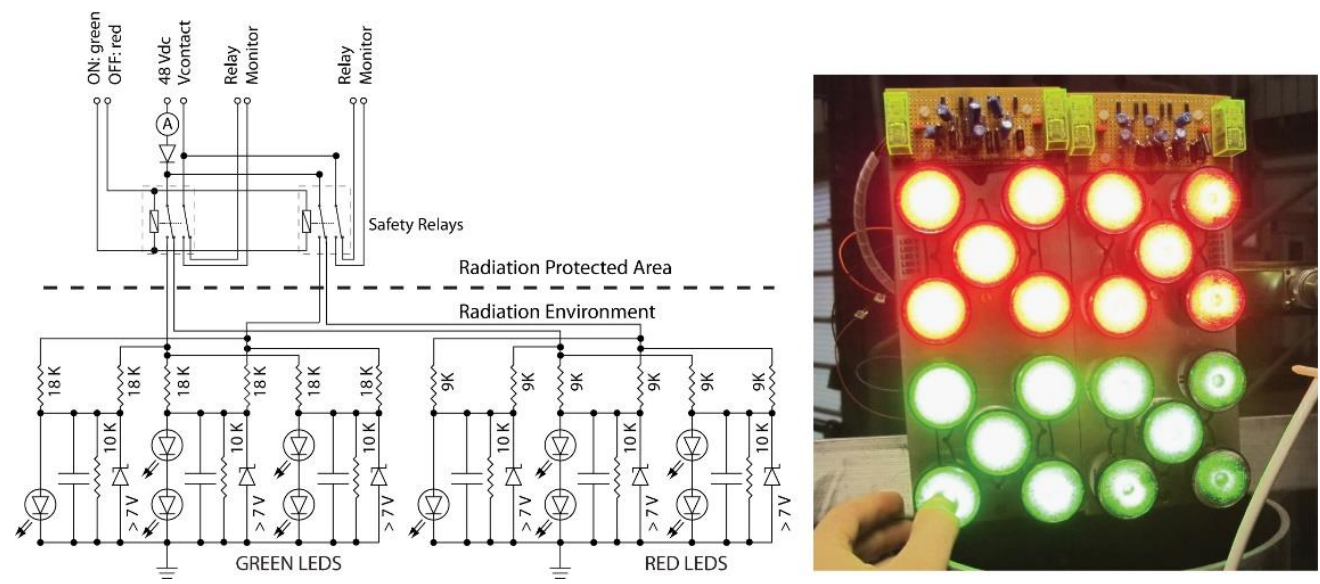

Figure 1: Electrical schematics and picture of the four cluster of five LEDs irradiated.

in the remote location and the corresponding light intensity reduction is negligible from a physiological point of view

The directional lightning shall be clearly visible from at least the tunnel line of sight that has a background luminance of about 4 to $6 \mathrm{~cd} / \mathrm{m} 2$ with brighter spots produced by the neon lighting and the emergency light. The luminance measurements rely on a Minolta LS-110 meter with an acceptance angle of $1 / 3^{\circ}$. Each red and green LED luminous flux is estimated to be respectively about $1.7 \mathrm{~lm}$ and $2 \mathrm{~lm}$.

The measurement of luminance assumes that the observation angle either encloses completely the light source or that the luminance is uniform on the observation area; at a distance of $10 \mathrm{~m}$ about $50 \%$ of the surface is not luminous and the measured luminance is underestimated by $50 \%$, figure 2 . At longer distances, the tunnel lights might perturb the meter.

The tests performed in the tunnel demonstrate that the directional lighting is clearly visible within a distance up to the maximum line of sight in the circular region. Within close proximity the luminance does not exceed $10^{\prime} 000 \mathrm{~cd} / \mathrm{m}^{2}$, which is an acceptable figure producing tolerable glare to personnel working in close proximity to the source of light [3].
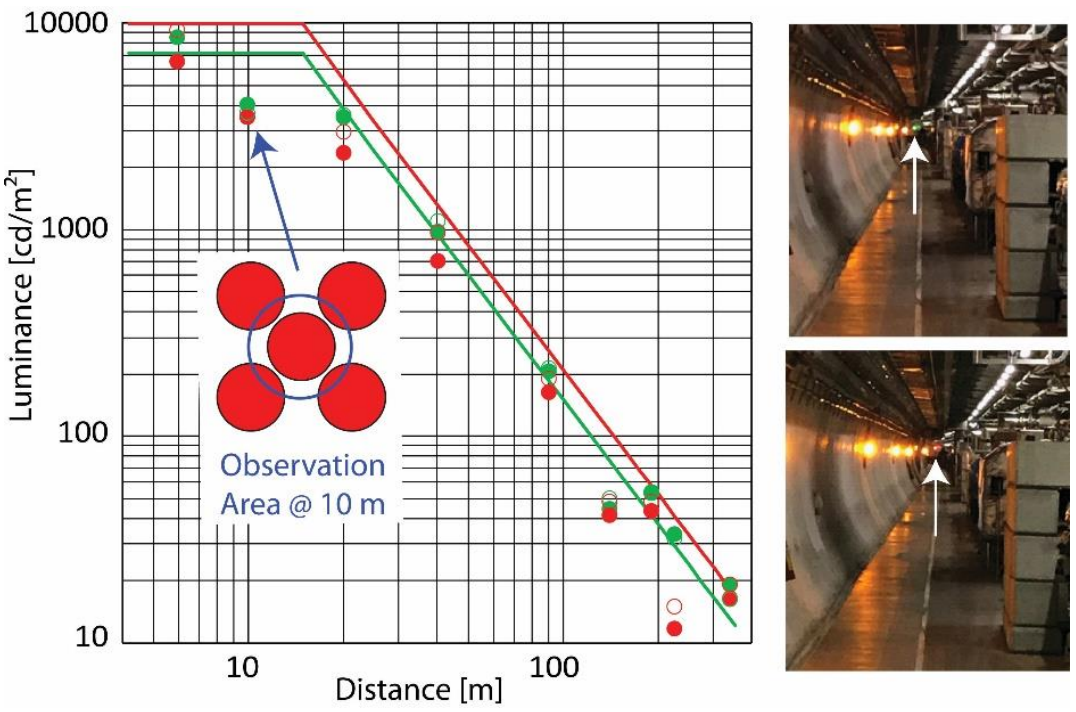

Figure 2: Measured (circles) and calculated (lines) luminance versus distance. Empty/Filled circles correspond to the clockwise/counter-clockwise direction. LCH tunnel view of the green and red directional lighting from a distance of $336 \mathrm{~m}$. 


\section{Radiation Tests}

The radiation tests were started in November 2014 in the CHARM irradiation facility on four clusters of five Cree XP-E2 red and green LEDs and their associated lens. Each cluster was powered independently and, during the irradiation, two were kept OFF. The irradiation campaign duration was 22 days and the Total Integrated Dose (TID) was 870 Gy. The luminance of each single lens and LED was measured at a distance of about $5.5 \mathrm{~m}$ corresponding to a viewing surface slightly smaller than the lens area when using the Minolta LS-110, figure 3.

There were five stops of the irradiation and the luminance was measured each time by the same person in the same geometrical conditions. The irradiation stops imposed a limited duration policy requiring to perform the measurements as fast as possible resulting in a large variation of the luminance data as seen on the relative luminance versus TID (figure 3).

Both red and green LEDs showed a decrease in luminance although this reduction in light intensity (about $80 \%$ for the red color) could not be recognized by the different persons that accessed the test area, it means that this reduction is not significant from a physiological point of view. Accidentally all LEDs were kept ON during two irradiation periods (grayed areas in figure 3).

The literature for radiation effects is mostly concerned with digital data transmission applications that typically use different light frequencies and different LED material. However some recent reports do exist for the same materials as used for the Cree XP-E2 LEDs. Blue LEDs made of InGaN are reported to decrease its luminance by about $18 \%$ and $47 \%$ for $340 \mathrm{keV}$ proton doses of respectively $1 \times 10^{12}$ and $5 \times 10^{12}$ proton/ $\mathrm{cm}^{2}$ [4]. The $\mathrm{InGaN}$ is the same technology used for the green Cree XP-E2 LED that during the CHARM test shows a decrease of about $30 \%$ for a dose of $870 \mathrm{~Gy}$ and $2.3 \times 10^{12} \mathrm{HEH} / \mathrm{cm}^{2}$. Up to a dose of $2 \times 10^{12} 20 \mathrm{MeV}$ proton/ $\mathrm{cm}^{2}$, the green LED ( InGaN) is almost insensitive to the radiation while the red LED (AlInGaP) shows a decrease of 30\% of its light output power [5]. Qualitatively the Cree XP-E2 radiation tests results are coherent with data reported in the litterature, although a quantitative comparison cannot be done because of different manufacturers for the LEDs and different radiation spectra.

At the end of the irradiation the PMMA material transparency was not affected by the accumulated radiation dose.

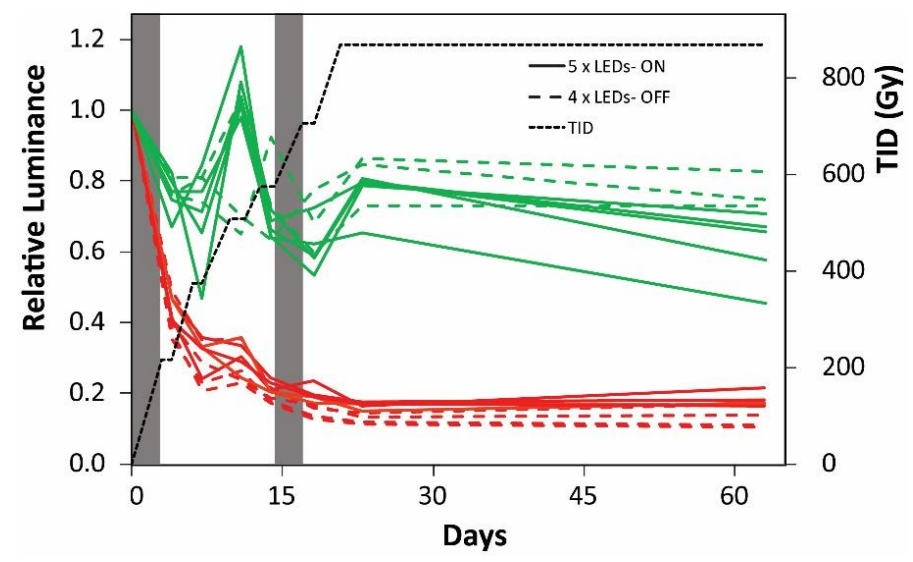

Figure 3: Luminance variation versus TID. Accidentally all LEDs were kept ON during the periods shown in gray 
After 2.5 years the lights were measured again, qualitatively the lights recovered about $20 \%$ of the loss of luminance (e.g. the green color was as bright as new) but the uncertainty of the measuring angle can easily yield errors of $50 \%$ on the measured luminance.

\section{Conclusions}

A directional lighting to permit or forbid personnel circulation in a given direction has been designed. Field tests confirm that the light intensity is compatible with good visibility over long distances in an environment like the LHC tunnel and has an acceptable amount of glare to personnel working in close proximity.

The present design provides ample robustness against failures like for instance parallel supplies of the LEDs circuitry to permit operation in case of LED failure, parallel cabling supplies to cope with open contacts, etc. A robust design requires that any degradation be observed, which can be performed by measuring the supply current that shall be able to detect open or shortened LEDs, open contacts, etc.

The TID does indeed produce a reduction of the LEDs luminance, but this reduction is not visible from a physiological point of view and if required the luminosity is easily increased by an increase of the supply current.

\section{Acknowledgements}

The authors are indebted to S. Grau for her technical comments concerning safety controls hardware and to F.J. Diez for his effort in preparing the prototypes and in performing field tests.

\section{References}

[1] L. Dufay-Chanat et al., Final report on the Controlled Cold Helium Spill Test in the LHC tunnel at CERN, Advances in Cryogenic Engineering: Cryogenic Engineering Conference, Tucson, AZ, USA 2015

[2] M. Green et al, Forensic Vision with Application to Highway Safety with CD-Rom 3rd Edition, Chapter 6.6 D Night visual performance, Vernon Odom Ph.D., Lawyers \& Judges, ISBN 978-1033264-54-3, 2008

[3] J. D. Bullough et al, Replacement Processes for Light Emitting Diode (LED) Traffic Signals, NCHRP Project 20-07/Task 246, 2009.

[4] S. J. Pearton et al, Review-Ionizing Radiation Damage Effects on GaN Devices, ECS Journal of Solid State Science and Technology, 5 (2) Q35-Q60 (2016).

[5] M. Boutillier, Protons effects on commercial European light emitting diodes, CNES-ESA presentation, 2015. 